\title{
SOURCE SigNAL STRENGTH INDEPENDENT LOCALIZATION
}

\author{
John R Rankin \\ Federation University Australia
}

\begin{abstract}
A problem with RSSI localization methods has been dependence on assumptions about the source signal strength or dependence on knowledge of its value at the source. This paper presents a solution to WSN localization for planar WSNs which averts this problem. It is a small, fine grained, range-based, beaconless, GPS-free, low cost RSSI technique which requires an irregular arrangement of four omnidirectional antennas for accurate positioning of the source and also for the determination of the source signal strength at the source. A spiral arrangement of antennas is described and software simulation of the localization process is presented that demonstrates its accuracy. Five omnidirectional antennas are fully sufficient to enable the avoidance of blind spots.
\end{abstract}

\section{KEYWORDS}

WSN (Wireless Sensor Network), sensor node, RSSI, localization.

\section{INTRODUCTION}

The problem of determining the position of radio sources relative to a given detector location with small low cost antennas is a continuing problem for useful data collection in mobile ad hoc networks (MANETs) and WSNs [1, 2, 3, 4]. If the distances between all nodes in the network can be measured by one of these techniques then a distance matrix can be formed and using the Map Maker algorithm [6] the coordinates of all the nodes are known up to a system translation, rotation or inversion. The hardware of the location detector needs to be small, portable, low cost and unobtrusive and yet also sufficiently accurate in determining positions [3]. It also needs to be low in the consumption of battery energy [12]. Directional antennas [7 to 11] do not match these requirements and do not allow for the free placement of nodes or moving nodes. Omnidirectional antennas are selected in this work for their low cost, simplicity as well as meeting the other criteria: a monopole antenna is more compact being half the size of a dipole antenna tuned to the same frequency. Omnidirectional antennas give us RSSI readings and therefore node to node distance measurements based on assumptions about $\psi_{0}$. Distance measurements can be obtained from RSSI measurements using the inverse square law which in suitable intensity and distance units is [5]

$$
\psi(r)=\frac{\psi_{0}}{r^{2}}
$$

Recently a multi-antenna technique [5] was published which only required the use of three omnidirectional antennas and the solution of two simultaneous linear inhomogeneous equations in two unknowns for the determination of relative coordinates. However these RSSI techniques require assumptions about the signal source intensity $\psi_{0}$ such as all nodes in the network having the same value of $\psi_{0}$ or else the $\psi_{0}$ values of all nodes are locally detected and broadcast to all nodes. The latter assumes that all nodes have RSSI detectors but this is not a low cost solution. Alternatively a node might know its own $\psi_{0}$ based on its own battery level and transmit this.

DOI: $10.5121 /$ ijwmn.2017.9403 
However it was also shown in [5] that measuring battery levels is also not a cost effective solution. A different approach is presented in this paper enabling any node with the localizator described in this paper to determine the relative positions of all other nodes, their distances and their local signal intensities $\psi_{0}$. In section 5 we discuss the application of the spiral array localizator to a 2 level balanced WSN [12].

\section{Distance Measurement With An Omnidirectional Antenna ARRAY}

When a wireless node is transmitting data its antenna produces a signal strength of EMR (ElectroMagnetic Radiation) which drops off according to the inverse square law given above (1). For the localization process we require that only one node of the network is transmitting at a time. A node located at point $\mathrm{A}$ in a WSN with an array of $\mathrm{n}$ simple omnidirectional antennas located at points $A_{i}$ for $i=1$ to $n$ within radius $R$ of $A$ can measure RSSI signal strengths of a single radio source located at point $\mathrm{S}$ to obtain the $\mathrm{n}$ readings $\psi_{i}$ for $\mathrm{i}=1$ to $\mathrm{n}$. This leads to $\mathrm{n}$ simultaneous quadratic equations for the relative coordinates $\left(\mathrm{x}_{\mathrm{S}}, \mathrm{y}_{\mathrm{S}}\right)$ of the source node $\mathrm{S}$ :

$$
\left(x_{S}-A_{i x}\right)^{2}+\left(y_{S}-A_{i y}\right)^{2}=\frac{\psi_{0}}{\psi_{i}}
$$

where $\psi_{0}$ is the RSSI of the source at unit distance from S. It will be assumed that $R^{2}<<\psi_{0} / \psi_{i}$ for all i. Since $\psi_{0}$ is not necessarily precisely known, we eliminate $\psi_{0}$ by dividing the i'th quadratic equation by the first quadratic equation for $\mathrm{i}=2$ to $\mathrm{n}$ thereby reducing the system to $\mathrm{n}-1$ quadratic equations. Further eliminating the quadratic quantity

$$
r_{S}^{2}=x_{S}^{2}+y_{S}^{2}
$$

reduces the system of equations to $\mathrm{n}-2$ simultaneous linear homogeneous equations for $\mathrm{x}_{\mathrm{S}}$ and $\mathrm{y}_{\mathrm{S}}$ which are then easily solved. Since we only need 2 such equations it follows that $n=4$ omnidirectional antennas are needed to construct this localizator. This results in the following equations

$$
\begin{aligned}
& a_{1} x_{S}+b_{1} y_{S}=c_{1} \\
& a_{2} x_{S}+b_{2} y_{S}=c_{2}
\end{aligned}
$$

where

$$
\begin{aligned}
& a_{1}=\left\{\frac{\left(A_{3 x}-\alpha_{3} A_{1 x}\right)}{\left(1-\alpha_{3}\right)}-\frac{\left(A_{2 x}-\alpha_{2} A_{1 x}\right)}{\left(1-\alpha_{2}\right)}\right\} \\
& a_{2}=\left\{\frac{\left(A_{4 x}-\alpha_{4} A_{1 x}\right)}{\left(1-\alpha_{4}\right)}-\frac{\left(A_{2 x}-\alpha_{2} A_{1 x}\right)}{\left(1-\alpha_{2}\right)}\right\} \\
& b_{1}=\left\{\frac{\left(A_{3 y}-\alpha_{3} A_{1 y}\right)}{\left(1-\alpha_{3}\right)}-\frac{\left(A_{2 y}-\alpha_{2} A_{1 y}\right)}{\left(1-\alpha_{2}\right)}\right\} \\
& b_{2}=\left\{\frac{\left(A_{4 y}-\alpha_{4} A_{1 y}\right)}{\left(1-\alpha_{4}\right)}-\frac{\left(A_{2 y}-\alpha_{2} A_{1 y}\right)}{\left(1-\alpha_{2}\right)}\right\}
\end{aligned}
$$




$$
\begin{aligned}
& c_{1}=\frac{\beta_{2}}{2\left(1-\alpha_{2}\right)}-\frac{\beta_{3}}{2\left(1-\alpha_{3}\right)} \\
& c_{2}=\frac{\beta_{2}}{2\left(1-\alpha_{2}\right)}-\frac{\beta_{4}}{2\left(1-\alpha_{4}\right)} \\
& \alpha_{1}=1 \\
& \alpha_{2}=\frac{r_{2}{ }^{2}}{r_{1}{ }^{2}}=\alpha_{1} \frac{\psi_{1}}{\psi_{2}} \\
& \alpha_{3}=\frac{r_{3}{ }^{2}}{r_{1}{ }^{2}}=\alpha_{1} \frac{\psi_{1}}{\psi_{3}} \\
& \alpha_{4}=\frac{r_{4}{ }^{2}}{r_{1}{ }^{2}}=\alpha_{1} \frac{\psi_{1}}{\psi_{4}} \\
& \beta_{2}=\alpha_{2} A_{1 x}{ }^{2}-A_{2 x}{ }^{2}+\alpha_{2} A_{1 y}{ }^{2}-A_{2 y}{ }^{2} \\
& \beta_{3}=\alpha_{3} A_{1 x}{ }^{2}-A_{3 x}{ }^{2}+\alpha_{3} A_{1 y}{ }^{2}-A_{3 y}{ }^{2} \\
& \beta_{4}=\alpha_{4} A_{1 x}{ }^{2}-A_{4 x}{ }^{2}+\alpha_{4} A_{1 y}{ }^{2}-A_{4 y}{ }^{2}
\end{aligned}
$$

So the solution is:

$$
\begin{aligned}
& x_{S}=\frac{b_{2} c_{1}-b_{1} c_{2}}{a_{1} b_{2}-a_{2} b_{1}} \equiv \frac{\text { detx }}{\text { det }} \\
& y_{S}=\frac{a_{1} c_{2}-a_{2} c_{1}}{a_{1} b_{2}-a_{2} b_{1}} \equiv \frac{\text { dety }}{\text { det }}
\end{aligned}
$$

Having found the position $\left(\mathrm{x}_{\mathrm{S}}, \mathrm{y}_{\mathrm{S}}\right)$ of the radio source by equations (2), (3), (4), (5) and (6), its strength at the source is found by:

$$
\psi_{0}=\psi_{1} r_{1}^{2}
$$

where

$$
r_{1}^{2}=x_{S}^{2}-2 A_{1 x} x_{S}+A_{1 x}{ }^{2}+y_{S}{ }^{2}-2 A_{1 y} y_{S}+A_{1 y}{ }^{2}
$$

If the antennas are placed symmetrically about the node A we would have:

$A_{1 x}=b, A_{1 y}=0$

$A_{2 x}=0, A_{2 y}=b$

$A_{3 x}=-b, A_{3 y}=0$

$A_{4 x}=0, A_{4 y}=-b$

where $b$ is a manufacture set value such that the radius of the localizator $R=b$ is less than the minimum node separation of the WSN. When substituted into equation (5) we get 


$$
\begin{aligned}
& \beta_{2}=\left(\alpha_{2}-1\right) b^{2} \\
& \beta_{3}=\left(\alpha_{3}-1\right) b^{2} \\
& \beta_{4}=\left(\alpha_{4}-1\right) b^{2}
\end{aligned}
$$

When substituted into equation (3) we get

$$
\begin{aligned}
& a_{1}=\left\{\frac{\alpha_{2}}{\left(1-\alpha_{2}\right)}-\frac{\left(1+\alpha_{3}\right)}{\left(1-\alpha_{3}\right)}\right\} b \\
& b_{1}=-\frac{b}{\left(1-\alpha_{2}\right)} \\
& a_{2}=\left\{\frac{\alpha_{2}}{\left(1-\alpha_{2}\right)}-\frac{\alpha_{4}}{\left(1-\alpha_{4}\right)}\right\} b \\
& b_{2}=-\left\{\frac{1}{\left(1-\alpha_{4}\right)}+\frac{1}{\left(1-\alpha_{2}\right)}\right\} b \\
& c_{1}=0 \\
& c_{2}=0
\end{aligned}
$$

This results in detx $=\operatorname{dety}=\operatorname{det}=0$ so that the equations (6) become indeterminate. To avoid this situation the antennas can be placed non-symmetrically about the node at A and a suitable antenna array for this is to use the arithmetic spiral so that

$$
\begin{aligned}
& A_{1 x}=b, A_{1 y}=0 \\
& A_{2 x}=0, A_{2 y}=2 b \\
& A_{3 x}=-3 b, A_{3 y}=0 \\
& A_{4 x}=0, A_{4 y}=-4 b
\end{aligned}
$$

A graphics program was written to test and demonstrate this spiral 4 antenna localizator method. The result of testing was that the spiral array of 4 antennas accurately localizes the source S. Typical results are shown in Figure 1 below. The black dashed lines show the signals travelling from $\mathrm{S}$ to each of the 4 antennas of the localizator. The black + sign shows where the radio source $\mathrm{S}$ was located on the grid by mouse click and the black circle shows the grid point where equations (2) to (6) above determined that the point $S$ is on the grid. It is clear from the program that the equations (2) to (6) give a very accurate estimate of the source position. The result of applying equations (7) and (8) to compute the local radio source signal strength $\psi_{0}$ is also shown below the main menu on the program window: the display on the window "psi0 $=\ldots$ " shows the accuracy of the computed $\psi_{0}$ compared with the actual $\psi_{0}$ where in the program the actual value of $\psi_{0}$ was set as exactly 100. It is clear from the program screen shot of Figure 1 that equation (7) gives $\psi_{0}$ accurately to 11 decimal places in these experiments and the program therefore shows that the equations above return a very accurate estimate of $\psi_{0}$. 


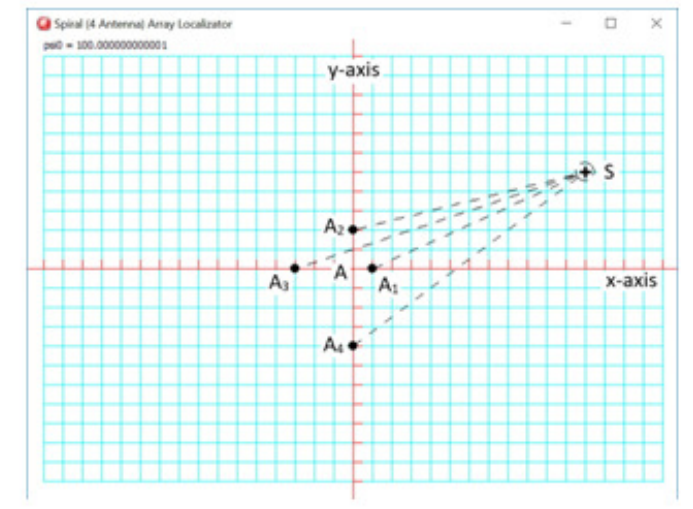

Figure 1. The spiral localizator consists of 4 antennas $A_{1}, A_{2}, A_{3}$ and $A_{4}$ positioned around a node $A$. It can measure the position of radio source at $S$.

\section{BLIND SPOTS}

The question that next arises is: Are there any source positions $S$ for which the above calculations fail? Firstly $a_{1}, b_{1}$ and $c_{1}$ cannot be calculated if $\alpha_{2}$ or $\alpha_{3}$ equals 1 . This occurs when either $r_{2}=r_{1}$ or $r_{3}=r_{1}$. Secondly $a_{2}, b_{2}$ and $c_{2}$ cannot be calculated if $\alpha_{2}$ or $\alpha_{4}$ equals 1 . This occurs when either $r_{2}=r_{1}$ or $r_{4}=r_{1}$. Thirdly $x_{S}$ and $y_{S}$ cannot be computed if $a_{1} b_{2}=a_{2} b_{1}$.

$$
\begin{aligned}
& a_{1} b_{2}-a_{2} b_{1}=\left\{\frac{\left(A_{3 x}-\alpha_{3} A_{1 x}\right)}{\left(1-\alpha_{3}\right)}-\frac{\left(A_{2 x}-\alpha_{2} A_{1 x}\right)}{\left(1-\alpha_{2}\right)}\right\}\left\{\frac{\left(A_{4 y}-\alpha_{4} A_{1 y}\right)}{\left(1-\alpha_{4}\right)}-\frac{\left(A_{2 y}-\alpha_{2} A_{1 y}\right)}{\left(1-\alpha_{2}\right)}\right\} \\
& -\left\{\frac{\left(A_{4 x}-\alpha_{4} A_{1 x}\right)}{\left(1-\alpha_{4}\right)}-\frac{\left(A_{2 x}-\alpha_{2} A_{1 x}\right)}{\left(1-\alpha_{2}\right)}\right\}\left\{\frac{\left(A_{3 y}-\alpha_{3} A_{1 y}\right)}{\left(1-\alpha_{3}\right)}-\frac{\left(A_{2 y}-\alpha_{2} A_{1 y}\right)}{\left(1-\alpha_{2}\right)}\right\}
\end{aligned}
$$

This is the third component of the cross product of vectors $\underline{a}=\left(a_{1}, a_{2}, 0\right)$ and $\underline{b}=\left(b_{1}, b_{2}, 0\right)$. The $2 D$ vector $\left(A_{3 x}-\alpha_{3} A_{1 x}, A_{3 y}-\alpha_{3} A_{1 y}\right)$ is a vector from point $A$ to a point $P$ on the line $A_{1} A_{3}$ but not at point $A_{1}$ or point $A_{3}$. The vector a is a vector proportional to the vector $\underline{A P}$. Likewise the $2 \mathrm{D}$ vector $\left(A_{4 x}-\alpha_{4} A_{1 x}, A_{4 y}-\alpha_{4} A_{1 y}\right)$ is a vector from point $A$ to a point $Q$ on the line $A_{1} A_{4}$ but not at point $A_{1}$ or point $A_{4}$. The vector $\underline{b}$ is a vector proportional to the vector $A Q$. The line AQ is the vertical $y$-axis and line AP is non-parallel to AQ. Therefore the cross product of $\underline{a}$ and $\underline{b}$ is never zero and so this third case never arises.

A way to eliminate the blind spots is to add three more antennas $A_{2}{ }^{\prime}, A_{3}{ }^{\prime}$ and $A_{4}{ }^{\prime}$. These extra antennas could conveniently be placed on the arithmetic spiral of points $A_{1}, A_{2}, A_{3}, A_{4}$ such that $A_{2}$ ' is midway between $A_{1}$ and $A_{2}, A_{3}$ ' is midway between $A_{2}$ and $A_{3}$ and $A_{4}{ }^{\prime}$ is midway between $\mathrm{A}_{3}$ and $\mathrm{A}_{4}$. The purpose of these extra antennas is to supply up to 3 extra signal strength readings $\psi_{2}{ }^{\prime}, \psi_{3}{ }^{\prime}, \psi_{4}{ }^{\prime}$. So if we have readings where $\psi_{2} \approx \psi_{1}$ then we can replace $\psi_{2}$ with $\psi_{2}{ }^{\prime}$ or if we have readings where $\psi_{3} \approx \psi_{1}$ then we can replace $\psi_{3}$ with $\psi_{3}{ }^{\prime}$ or if we have readings where $\psi_{4} \approx \psi_{1}$ then we can replace $\psi_{4}$ with $\psi_{4}^{\prime}$ 'so that we will have equations without the detected blind spot. This placement of the extra antennas means that the localizator is still compact. While we could have any number of antennas on the localizator and they could be arranged on an arithmetic spiral of many turns the result could be a localizator which is too big, bulky and intrusive in the network especially when many nodes need such localizators. The maximum 
radius enclosing a localizator, $\mathrm{R}$, should be significantly less than the distance to the nearest other node of the WSN and for dense WSNs we therefore need compact localizators. An advantage of the spiral array of antennas is that the normals to the spiral curve always intersect closer to the centre of the spirals than the points on the spiral where the normal is taken. The same occurs for the perpendicular bisectors of chords of the spiral. By having the maximum radius enclosing a localizator $\mathrm{R}$ far less than the distance between the minimum node separation we therefore cannot have double blind spots or triple blind spots occurring. This means that blind spots are caused by only one pair of antennas and not more. Figure 2 below shows the blind spots for antennas at $A_{1}$, $A_{2}, A_{3}$ and $A_{4}$ as any points on the green lines. Since blind spots can only be single, the extra antennas $A_{2}{ }^{\prime}, A_{3}$ ' and $A_{4}$ ' can be cut back to a single antenna at $A_{5}$ positioned at $(2 b, 0)$. Figure 2 also shows the blind spots for the antennas $\mathrm{A}_{5}, \mathrm{~A}_{2}, \mathrm{~A}_{3}$ and $\mathrm{A}_{4}$ as purple lines. The purple lines do not intersect the green lines outside a distance of the maximum radius $\mathrm{R}$ enclosing the localizator at $\mathrm{A}$ and therefore a source location $\mathrm{S}$ cannot simultaneously be a blind spot for the antenna array $\mathrm{A}_{1}, \mathrm{~A}_{2}, \mathrm{~A}_{3}, \mathrm{~A}_{4}$ and also the antenna array $\mathrm{A}_{5}, \mathrm{~A}_{2}, \mathrm{~A}_{3}, \mathrm{~A}_{4}$. Therefore if the raw measurements $\psi_{2}, \psi_{3}, \psi_{4}$ have any one equal with $\psi_{1}$ indicating the presence of a blind spot then $\psi_{5}$ will be used in place of $\psi_{1}$ and we are assured by the above reasoning that no equal with $\psi_{5}$ will occur, i.e. that no blind spot will now occur. This also causes minimal changes to the equations to be computed since only $A_{1 x}$ is changed to $A_{5 x}$ and $\alpha_{1}$ should be changed from $\alpha_{1}=1$ to $\alpha_{1}=\frac{\psi_{5}}{\psi_{1}}$ and no other terms in the equations above change. This leads to the following algorithm for the localizator.

\section{SPIRAL LOCALIZATOR AlgORITHM}

A spiral localizator at a node at position A consists of 5 antennas irregularly but accurately placed around the node at positions $\mathrm{A}_{1}, \mathrm{~A}_{2}, \mathrm{~A}_{3}, \mathrm{~A}_{4}$ and $\mathrm{A}_{5}$. To accurately determine the position of a radio source $S$ all other radio sources (at the given frequency) must be turned off and then the strength of the radio signal from $\mathrm{S}$ is measured at the 5 antennas as $\psi_{1}, \psi_{2}, \psi_{3}, \psi_{4}, \psi_{5}$. From these 5 raw measured values the planar position of a radio source $\left(\mathrm{x}_{\mathrm{S}}, \mathrm{y}_{\mathrm{S}}\right)$ and its local signal strength $\psi_{0}$ are determined by the following spiral localizator algorithm. The spiral localizator has the following given constants:

$$
\begin{aligned}
& A_{1 x}=b, A_{1 y}=0 \\
& A_{2 x}=0, A_{2 y}=2 b \\
& A_{3 x}=-3 b, A_{3 y}=0 \\
& A_{4 x}=0, A_{4 y}=-4 b \\
& A_{5 x}=2 b, A_{5 y}=0
\end{aligned}
$$

where $b$ is a characteristic size constant of the localizator antenna array. 


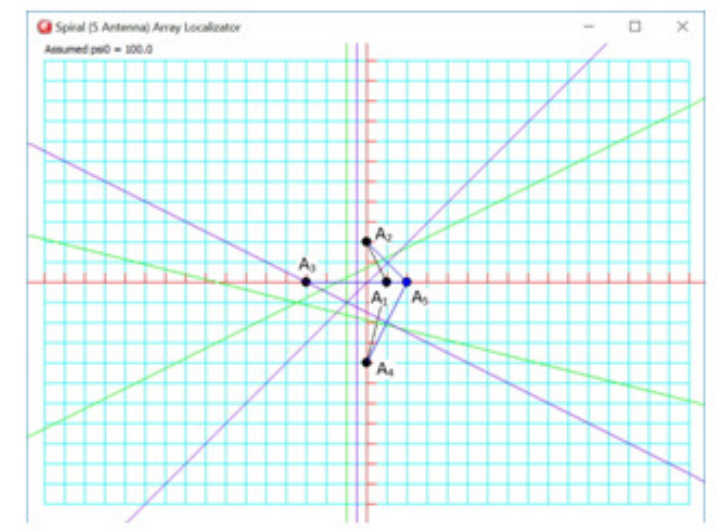

Figure 2. Blind spots are shown as green lines for antennas $A_{1}, A_{2}, A_{3}$ and $A_{4}$ and as purple lines for antennas $\mathrm{A}_{5}, \mathrm{~A}_{2}, \mathrm{~A}_{3}$ and $\mathrm{A}_{4}$.

Obtain the raw data $\psi_{1}, \psi_{2}, \psi_{3}, \psi_{4}, \psi_{5}$ from each of the 5 antennas. If $\psi_{1}$ is different from $\psi_{2}$ and from $\psi_{3}$ and from $\psi_{4}$ then compute $\alpha_{2}, \alpha_{3}, \alpha_{4}$ from equations (4). Next compute $\beta_{2}, \beta_{3}, \beta_{4}$ from equations (9) and (8). Next compute $a_{1}, a_{2}, b_{1}, b_{2}, c_{1}, c_{2}$ from equations (3) and then $\mathrm{x}_{\mathrm{S}}$ and $\mathrm{y}_{\mathrm{S}}$ from equation (6). Then compute $\mathrm{r}_{1}$ from equation (8) and finally $\psi_{0}$ from equation (7).

However if $\psi_{1}$ is equal to either $\psi_{2}$ or $\psi_{3}$ or $\psi_{4}$ then use

$$
\begin{aligned}
& A_{1 x}=2 b, A_{1 y}=0 \\
& A_{2 x}=0, A_{2 y}=2 b \\
& A_{3 x}=-3 b, A_{3 y}=0 \\
& A_{4 x}=0, A_{4 y}=-4 b
\end{aligned}
$$

and replace $\alpha_{1}=1$ with $\alpha_{1}=\frac{\psi_{5}}{\psi_{1}}$ to compute $\alpha_{2}, \alpha_{3}, \alpha_{4}$ from (4) or simply use $\alpha_{2}=\frac{\psi_{5}}{\psi_{2}}$ $\alpha_{3}=\frac{\psi_{5}}{\psi_{3}}$ $\alpha_{4}=\frac{\psi_{5}}{\psi_{4}}$

Next compute $\beta_{2}, \beta_{3}, \beta_{4}$ from equations (9) and (8). Next compute $a_{1}, a_{2}, b_{1}, b_{2}, c_{1}, c_{2}$ from equations (3) and then $\mathrm{x}_{\mathrm{S}}$ and $\mathrm{y}_{\mathrm{S}}$ from equation (6). Then compute $\mathrm{r}_{1}$ from equation (8) and finally $\psi_{0}$ from equation (7).

Then the radio source is located at $\mathrm{S}=\left(\mathrm{x}_{\mathrm{S}}, \mathrm{y}_{\mathrm{S}}\right)$ with signal intensity $\psi_{0}$. A graphics program was written to implement this algorithm and results showing the two paths of the algorithm are shown in Figures 3 and 4 below. It was observed that in all cases the algorithm correctly avoided blind spots and accurately returned $\mathrm{x}_{\mathrm{S}}, \mathrm{y}_{\mathrm{S}}$ and $\psi_{0}$. 
It is clear that the spiral localizator algorithm can be coded in software that runs on every node that has a localizator, or else to save computational time and battery consumption the algorithm can be cheaply encoded in combinational hardware chips in the localizator itself with the output readable to the local node which has the localizator. A third possibility is to send the raw data $\left\{\psi_{1}, \psi_{2}, \psi_{3}, \psi_{4}, \psi_{5}\right\}$ up the line to the next level for computation in software assuming that the next level has greater power and computation capability. Indeed the raw data can be relayed up the line all the way to the mains powered base station which also has more capability than the nodes of the WSN.

\section{DISCUSSION}

A graphics program was written to test the accuracy of the 4 antenna spiral localizator. The output is seen in Figure 1. No matter where the source node $\mathrm{S}$ is selected on the grid shown, the coordinates $\left(\mathrm{x}_{\mathrm{S}}, \mathrm{y}_{\mathrm{S}}\right)$ are accurately computed and shown graphically. However we can also notice that no grid positions occur on the green lines which are where the blind spots lie. A second program therefore was developed to allow any point off the grid to be selected as the source $S$ and this program uses the algorithm of section 4 above to avoid blind spots - see Figure 3 . When $A_{5}$ is not used in the algorithm, the black dot for $A_{1}$ is shown and the blue dot for $A_{5}$ is not shown in the graphical display. When $A_{5}$ is used in the algorithm the blue dot for $A_{5}$ is shown and the black dot for $\mathrm{A}_{1}$ is not shown in the graphical display. The results showed that the algorithm correctly changed the input raw data from $\left\{\psi_{1}, \psi_{2}, \psi_{3}, \psi_{4}\right\}$ to $\left\{\psi_{5}, \psi_{2}, \psi_{3}, \psi_{4}\right\}$ in order to avoid blind spots. It also shows high accuracy in computing the source position and the source signal strength $\psi_{0}$.

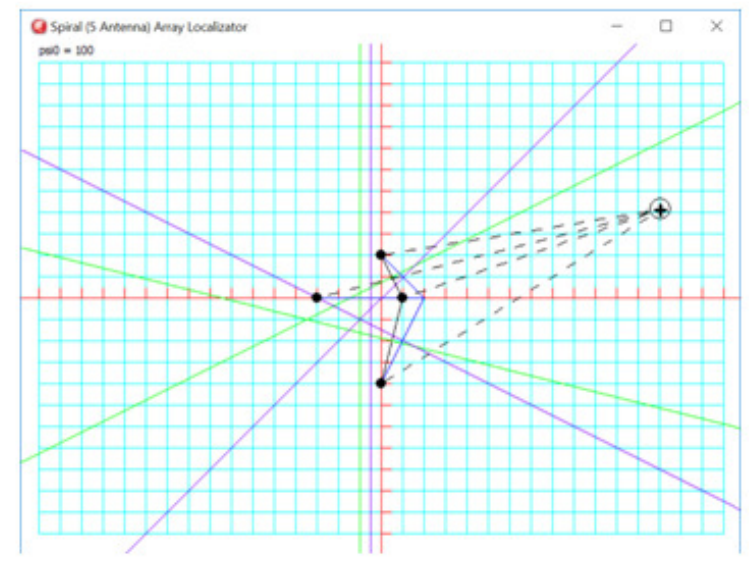

Figure 3 showing the Spiral Localizator algorithm using raw data $\left\{\psi_{1}, \psi_{2}, \psi_{3}, \psi_{4}\right\}$.

We now discuss the application of the localizator described in this paper to the general 2-level balanced WSN [12]. In this type of WSN we have data collected by $\mathrm{N}_{1}$ clusters each of $\mathrm{N}_{2}$ sensor nodes. The sensor nodes transmit their data to their respective cluster heads called aggregator nodes, and the $\mathrm{N}_{1}$ aggregator nodes relay this data on to the base station. In the WSN there are therefore $1+\mathrm{N}_{1} \mathrm{~N}_{2}$ nodes in total but not all nodes need localizators. As discussed in [5], only the base station and aggregators are provided with localizators. Each 5 antenna spiral localizator defines the $\mathrm{x}$ and $\mathrm{y}$ axial directions since it has antennas 1, 3 and 5 along the local $\mathrm{x}$-axis and antennas 2 and 4 along the local y-axis. All the localizators in a WSN can be aligned when their corresponding nodes are placed by turning them in the horizontal plane so that the line of 
antennas 1, 3 and 5 points to a given distant landmark by line of sight indicating the direction of the $\mathrm{x}$-axis and this is then checked with antennas 2 and 4 pointing to a different distant landmark by line of sight indicating the direction of the y-axis. As explained in [5] it is not necessary for the local axes of each localizator to be aligned since when all the localization data is transmitted up to the base station, the base station makes the computations to determine the relative orientations of the $\mathrm{x}$ and $\mathrm{y}$-axes of every aggregator localizator with respect to the orientation of the base station localizator.

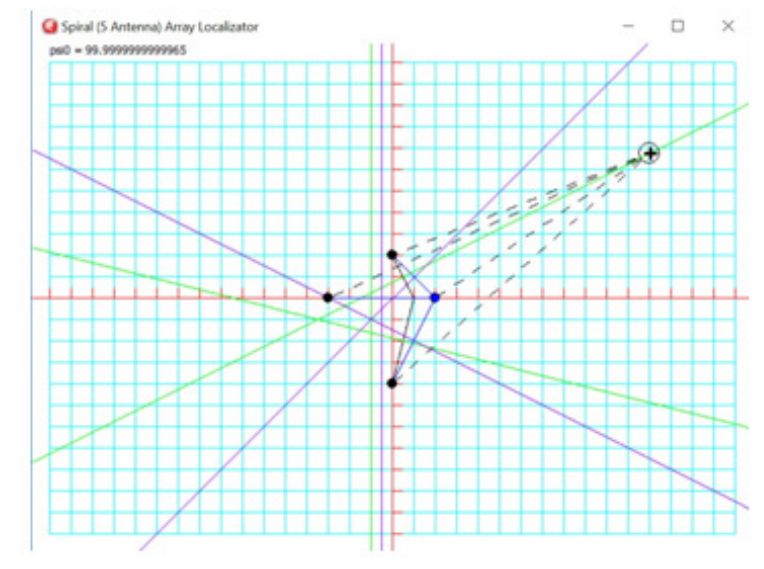

Figure 4 showing the Spiral Localizator algorithm using raw data $\left\{\psi_{5}, \psi_{2}, \psi_{3}, \psi_{4}\right\}$ to remove a blind spot.

The localization process for WSNs requires that the normal data collection operations of all nodes cease until the localization process of the whole WSN is completed to avoid radio interference in the measurement process though it would be possible for non-radio overlapping clusters to localize separately and in parallel. During the localization process only one sensor node may transmit a test pattern at a time for the duration required for the localizator of its aggregator node to capture the required raw data $\left\{\psi_{1}, \psi_{2}, \psi_{3}, \psi_{4}, \psi_{5}\right\}$ of that source. Likewise when the base station uses its localizator to localize an aggregator node all other aggregator nodes (as well as all sensor nodes) must remain off. For a dense 2-level balanced WSN, radio silence must be observed by all nodes of the WSN. In [5] a dense WSN was defined as one where full connectivity is initially possible. This means that the maximum separation of any two nodes in the WSN is less than the initial radius of radio reach of each node of say $\rho=10 \mathrm{~m}$. If all nodes of the WSN are within radio reach of the base station, then only the base station needs a localizator and aggregator and sensor nodes can be identical so that each has the same battery capacity. With no energy draining ADC chips in any node (including no battery level measuring hardware) and no localizators at any node, node lifetimes are maximized and node costs are minimized. With the only localizator located at the mains powered base station all localization computations are done at the base station and no localization computations are made at the nodes. In this case there is only one local coordinate system so no alignment of localizators is required and no computations compensating for non-aligned localizators is necessary. How long this ideal arrangement of radio coverage would last depends on the density of the WSN since all battery operated signals will eventually attenuate and it could be that having localizators at every aggregator node may result in a WSN with longer lifetime. There is therefore a trade-off between the operational longevity of the WSN and its cost in terms of the number of localizators installed. 
International Journal of Wireless \& Mobile Networks (IJWMN) Vol.9, No.4, August 2017

\section{Conclusions}

Whereas other localizators have assumed a value for the signal intensity at a radio source in order to compute its position, an array of antennas and corresponding algorithm has now been found that computes the location of a radio source $S$ and also calculates the signal intensity at $S$. This new localizator uses a compact arrangement of no more than 5 omnidirectional antennas. It provides a cheaper means of WSN localization than using directional antennas to determine angles and distances. The cheaper and simpler omnidirectional antennas can be used for network localization provided each localizator has at least 5 omnidirectional antennas in manufacturer fixed positions relative to the node. The equations for the position $\left(\mathrm{x}_{\mathrm{S}}, \mathrm{y}_{\mathrm{S}}\right)$ of a radio source are linear and therefore quick to compute. The formulas for computing $\left(\mathrm{x}_{\mathrm{S}}, \mathrm{y}_{\mathrm{S}}\right)$ can also be put into the hardware circuitry for the localizator to speed up the localization process and reduce the computational burden and energy consumption of a battery-based node. Alternatively the aggregators can send their raw data $\left\{\psi_{1}, \psi_{2}, \psi_{3}, \psi_{4}\right\}$ or $\left\{\psi_{5}, \psi_{2}, \psi_{3}, \psi_{4}\right\}$ to the base station and the calculation of $\left(\mathrm{x}_{\mathrm{S}}, \mathrm{y}_{\mathrm{S}}\right)$, the coordinates of a node relative to an aggregator and the calculation of $\left(\mathrm{x}_{\mathrm{S}}, \mathrm{y}_{\mathrm{S}}\right)$, the coordinates of that same node relative to the base station, as well as the calculation of $\psi_{0}$ the signal strength at $\mathrm{S}$, are then all done on the more powerful base station PC. Note that not all nodes in the WSN need a localizator: only the base station and aggregator nodes require a localizator thereby reducing equipment costs. This method works even if nodes in the WSN can move freely as in ad hoc networks, or if node radio signals are subject to considerable attenuation as the battery discharges, so that not all nodes have to have localizators. This cuts down the cost of the WSN. Furthermore the ADCs for battery level readings should be disabled or removed from the nodes to further reduce the cost of the WSN. If the nodes are static then the localization process is only required once. If nodes are slowly moving then savings in the battery life of the aggregators can also be achieved by not executing the localization process too frequently. Further battery savings can be obtained by keeping $\psi_{0}$ for all sensor nodes just above the level necessary for error free detection by the aggregators.

\section{REFERENCES}

[1] Z Luo, P Min, "Survey of Target Localization Methods in Wireless Sensor Networks", 2013 19th IEEE International Conference on Networks (ICON), 11-13 Dec, 2013.

[2] T Chowdhury, C Elkin, V Devabhaktuni, D Rawat, J Oluoch, “Advances On Localization Techniques For Wireless Sensor Networks: A Survey”, Computer Networks, Vol 110, pp 284-305, 9 Dec 2016.

[3] V Walia, S Kaur, "Survey of Different Localization Techniques of Wireless Sensor Networks”,IJEEE, Vol 1, Issue 4, pp 20-23, Aug 2014.

[4] N Alrajeh, M Bashir, B Shams, "Localization Techniques in Wireless Sensor Networks", International Journal of Distributed Sensor Networks, vol 2015, art 304628, 9 pp, 2013 Hindawi Publishing Corporation.

[5] J Rankin "Multi-antenna Localization Method", International Journal of Wirelesss and Mobile Networks", tba, 2017.

[6] J Rankin, "Map Making From Tables", International Journal of Computer Graphics and Animation, April 2013, Vol 3, Nr 2.

[7] M Nilsson, "Directional Antennas for Wireless Sensor Networks", Martin Nilsson. SICS, P.O.B.1263, SE-164 29 Kista, Sweden from DOT adhoc09 AT drnil DOT com, 2009.

[8] L Mottola, T Voigt, G Picco, "Electronically-switched Directional Antennas for Wireless Sensor Networks: A Full-Stack Evaluation", Sensor, Mesh and Ad Hoc Communications and Networks (SECON), 2013 10th Annual IEEE Communications Society Conference, June 2013. 
International Journal of Wireless \& Mobile Networks (IJWMN) Vol.9, No.4, August 2017

[9] G Tarter, L Mottola, G Picco, "Directional Antennas for Convergecast in Wireless Sensor Networks: Are They A Good Idea?”, University of Trento Italy, http://disi.unitn.it/ picco/papers/mass16.pdf

[10] G Whyte, “Antennas for Wireless Sensor Network Applications”, PhD thesis, Department of Electrical and Electronic Engineering, University of Glasgow, 2008.

[11] Q Wang, H Dai, Z Zheng, M Imran, A Vasilakos, "On Connectivity of Wireless Sensor Networks with Directional Antennas”, Sensors Journal, 17, 134, 22pp, 2017.

[12] J Rankin "Optimal Data Collection for a 2-Level Balanced WSN", International Journal of Wireless and Mobile Networks, Vol 9, No 3, 2017.

\section{AUTHOR}

J Rankin has undertaken research over 45 years starting with General Relativity and Mathematical Physics at the University of Adelaide followed by Computer Graphics, Fuzzy Logic Systems and Games Technology at La Trobe University and most recently Wireless Sensor Networks with Charles Sturt University Study Group Melbourne.

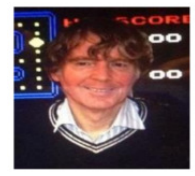

